\title{
Basic emotions expressed in music: factor analyses on intensity ratings by non-musical professional Chinese university students
}

This article was published in the following Dove Press journal:

Psychology Research and Behavior Management

\author{
Chanchan Shen' \\ Mufan Wang ${ }^{2}$ \\ Tongjun Ding ${ }^{3}$ \\ Yang Yang ${ }^{4}$ \\ Javier Cabanyes-Truffino ${ }^{5}$ \\ Lijun Sun ${ }^{6}$ \\ Chu Wang' \\ Wei Wang' \\ 'Department of Clinical Psychology \\ and Psychiatry, School of Public \\ Health, Zhejiang University College \\ of Medicine, Hangzhou, China; \\ ${ }^{2}$ Faculty of Psychology, Universidad \\ Complutense de Madrid, Madrid, \\ Spain; ${ }^{3}$ Department of Musicology, \\ Qianjiang College, Hangzhou \\ Normal University, Hangzhou, China; \\ ${ }^{4}$ Department of Musicology, College \\ of Arts and Communications, Anhui \\ University, Hefei, China; ${ }^{5}$ Faculty of \\ Education, Universidad Complutense \\ de Madrid, Madrid, Spain; ${ }^{6}$ CAS Key \\ Laboratory of Behavioral Science, \\ Institute of Psychology, Department \\ of Psychology, University of Chinese \\ Academy of Sciences, Beijing, China
}

Correspondence: Wei Wang Department of Clinical Psychology and Psychiatry, School of Public Health, Zhejiang University College of Medicine, Yuhangtang Road 866, Hangzhou, Zhejiang 310058 , China

$\mathrm{Tel}+8657188208188$

Fax +86 57। 8820 8I88

Email drwangwei@zju.edu.cn
Background: Previous studies of musical emotion largely depended on the lexical approach which suffered from overlaps between emotions.

Methods: In the present study, we explored emotional domains through a dimensional approach based on the intensity ratings on the emotion perceived in music. Altogether, 488 university students were invited to listen to 60 musical excerpts (most of them classical), to rate the intensity of emotion perceived without naming the emotion. Later, we conducted the exploratory factor analysis on the intensity ratings to look for the latent structures of musical emotion and then applied the confirmatory factor analysis to verify the validity of the proposed model of emotional structure. Results: After first- and second-order factor analyses, seven emotional factors (domains, with 38 musical excerpts) were identified: Happiness, Tenderness, Sadness, Passion, Anger, Anxiousness, and Depression, which formed a satisfactory model. No gender difference was found regarding the perceived intensity of musical emotion.

Conclusion: Our study has offered evidence to delineate basic musical emotions into seven domains. Keywords: dimensional classification, factor analysis, intensity rating, musical emotion

\section{Introduction}

Music plays an important role in everyday life, such as in commercial sites to stimulate consuming behaviors ${ }^{1}$ and in leisure activity to regulate individual emotion and mood. ${ }^{2}$ It is also used as a kind of psychotherapy for pain ${ }^{3}$ and other emotional problems. ${ }^{4}$ Indeed, when regarding processing or recognizing a putative emotion selected from a musical source, we are not sure that an emotion is specifically elicited by its putative vector, neither we are sure that an emotion is specifically recognized from its putative vector. Therefore, the music effectiveness is not ideal due to the imprecise application of desired emotional music, which results from the rough classification of emotion. The question of how many emotions people can perceive from music is still under debate, despite there being plenty of research on music and emotion. Many studies were focused on musical emotions which were not theoretically driven or not comprehensive, ${ }^{5,6}$ for example, researchers tended to select several emotions of interest (mostly happiness, sadness, and anger) for their designs. Given that music influences various fields including scientific research, education, and therapy, it is of vital theoretical and practical significance to acquire a comprehensive picture of what kinds of emotion are effectively conveyed by music.

There are several proposed models to depict musical emotions: the circumplex model, ${ }^{7}$ the positive activation-negative activation model, ${ }^{8,9}$ and the vector model. ${ }^{10}$ These models are mostly based on the planar quadrant distributions of arousal and 
valence of emotion expressed in music, but also have their own uniqueness. For instance, the circumplex model proposes that emotions are distributed in a circular pattern centered on medium arousal and neutral valence. The vector model illustrates an underlying dimension of arousal and a binary choice of valence that determines direction, which results in two vectors. Both vectors start at zero arousal and neutral valence and proceed as straight lines, one in a positive and the other one in a negative valence direction. Apparently, both vector model and the positive activation-negative activation model include the neutral valence and the high arousal ratings, which result from the averaging of individual positive and negative valence ratings; therefore, they provide overall better fits of emotional classification than the circumplex model does. ${ }^{9}{ }^{11}$ However, the two-dimensional models are not ideally independent, since the higher arousal states are more likely to be defined by valence and the lower arousal states are more likely to be neutral. ${ }^{11}$ Practically, in a human neurophysiological study, the magnitudes of the event-related skin conductance responses varied according to different musical emotions, but did not parallel with subjects' ratings on their arousal levels. ${ }^{12}$ Nevertheless, the multiple-dimensional method based on the factor analyses might be a better way to classify musical emotions.

In parallel, when referring to the types of emotion conveyed by music, most previous approaches to delineating musical emotions were taxonomical (lexical), that is, to characterize these emotions with terms. For instance, Hevner developed a celebrated, music-specific emotion model, including eight emotional clusters (ie, Sober, Gloomy, Longing, Lyrical, Sprightly, Joyous, Restless, and Robust), each described with six to eleven emotion-colored adjectives. ${ }^{13}$ The eight groups were arranged into a circle, so that the adjacent clusters possessed certain features in common, while the clusters in the opposite location deviated mostly from each other. This musical emotion model was later refined and rearranged into 9-13 clusters and then sorted into six subgroups. ${ }^{14,15}$ Furthermore, in a study of updating the Hevner adjectives, nine emotional clusters were elaborated. ${ }^{16}$ This outcome was partly supported by Juslin and Laukka who studied every day listening and found nine clusters of emotion, labeled as joy, sadness, love, calm, anger, tenderness, longing, solemnity, and anxiety. ${ }^{17}$ Recently, Zentner et al compiled a comprehensive list of emotion-colored adjectives and asked participants to rate the frequency of the given emotions they perceived in their preferred music and in their everyday life. ${ }^{18}$ They performed the exploratory factor analysis (EFA) and confirmatory factor analysis (CFA) on the ratings and developed the Geneva Emotional Music Scale which consisted of nine factors: wonder, transcendence, tenderness, nostalgia, peacefulness, power, joyful activation, tension, and sadness.

Through the lexical approach on musical emotions, some emotions, such as happiness, anger, sadness, tenderness, and anxiety, were consistently recognized in children, in individuals of different cultures, and in brain-damaged or neuropsychiatric patients. ${ }^{19}$ However, these studies suffered from several shortcomings. Firstly, the semantic consideration was the sorting of emotion-colored adjectives which were suitable for describing rather than for classifying musical emotion. Meanwhile, this kind of classification was influenced by cultural information embedded into language. When it came to some languages without terms describing emotion, ${ }^{20}$ such a method would fail to generate a musical emotion-related structure. Secondly, the classification clusters were subjective; thus, the structure of musical emotion derived would lack structural validity. For instance, nouns with similar meanings, such as sorrow, sadness, and grief, were prudently classified into the same emotional family; ${ }^{21}$ thus, they might fail to capture nuanced emotional categories. Zentner et al, ${ }^{18}$ however, applied the EFA to the emotional terms, but they focused more on the music-induced emotions and reached unsatisfactory validities of those perceived emotions. Thirdly, the musical stimuli were not presented to participants in most studies, and participants reported their judgments which were greatly dependent on their memory and their past musical experiences.

The purpose of the present study was to delineate emotions conveyed in music, with two approaches. One was that we prepared a more comprehensive pool of musical excerpts (MATRIX, most of them were classical) which conveyed 13 categories of dominant musical emotions as proposed through a previous lexical study. ${ }^{15}$ The other was that we invited participants to rate only the intensity of the emotion they perceived (instead of naming that emotion) when listening to each of the MATRIX excerpts. The intensity ratings are measured with the Likert scale which is frequently used in psychological assessments, and produce multipledimensional structures of personality or other psychological measures. ${ }^{22,23}$ Then, we constructed and validated the basic structures of musical emotion by conducting both EFA and CFA on the intensity ratings. Actually, in this way, the investigators have successfully delineated the structures of facial expressions of emotion ${ }^{24}$ and picture vectors of emotion. ${ }^{25}$ In the current study, we have hypothesized that musical emotion at least includes happiness, tenderness, sadness, anger, 
and anxiousness and these emotions have their discriminant and convergent validities, since previous documentation had indicated that these emotions are the basic ones related to music. ${ }^{17,26}$ Moreover, we would like to test the gender difference, since it is well acknowledged that females are more sensitive to negative emotion than males are. ${ }^{27}$

\section{Materials and methods Participants}

Altogether, 488 university students who were not majoring in musicology and related specialties and were without previous musical training participated in the study: 214 males (mean age, 20.13 years with SD 1.51; age range, 16-28 years) and 274 females (mean age, 19.86 with SD 1.41; range, 17-28 years). All participants were with normal hearing, without any neurological or psychiatric disorders, and were free from drug or alcohol 72 hours prior to the test. The study protocol was approved by the Ethics Committee of Zhejiang University College of Medicine (No. ZGL201606-1-2), and all participants gave their written informed consents (the informed consents of the young adolescents were signed by their guardians).

\section{Likert scale}

Likert scale was used to investigate the intensity ratings of the perceived musical emotion from each participant. In the current study, we defined the scale with nine grades (from 0 to 8 ); the larger the number, the stronger the emotion a participant perceived. Zero indicates that the intensity of perceived emotion is very low or approaches to peace, 1 indicates the intensity is weak, 4 indicates it is moderate, and 8 indicates the strongest intensity.

\section{Musical excerpts and procedure}

In order to construct a musical database, we set up a list of emotional labels containing as many names of emotion as possible, which included 13 musical emotions (ie, sad, fanciful, frustrated, agitated, longing, dreamy, passionate, mysterious, scared, dramatic, delicate, bluesy, and happy) as proposed in a lexical study. ${ }^{15}$ Bearing these emotions in mind, 66 excerpts of nonvocal music, most of which were classical, were chosen by two well-experienced professors of musicology (also our coauthors: one $\mathrm{PhD}$ holder, TD; and one master's holder, YY).

Then, the preliminary 66 musical excerpts were presented to seven judges: the above-mentioned two musicology professors and other five of our co-authors (one $\mathrm{PhD}$ holder in musicology, LS; one PhD holder in psychiatry, WW; one MD candidate, $\mathrm{CS}$; one master's candidate in psychiatry, $\mathrm{CW}$; and one master's candidate in psychology, MW). The judges were asked to listen to the musical excerpts and to decide whether they conveyed significantly salient emotions. Each excerpt was voted on by seven judges independently. If an excerpt received more than four "no" votes, it was dismissed. If an excerpt received three "yes" and three "no" votes, the seventh judge (WW) made the final decision. Finally, 60 musical excerpts were retained to form a MATRIX. Table 1 illustrates the information about the MATRIX musical excerpts, and the related musical scores (texts) will be available upon request to the authors. The mean duration of these excerpts was 30.72 seconds (with SD 9.51) and ranged from 16 to 62 seconds, and the different durations were due to different needs for bearing salient emotions.

Figure 1 illustrates the general procedure of the experiment. Participants were led to a quiet room to have rest for 5 minutes. Later, they were explained that the present study was designed to delineate musical emotions. They were then asked to listen to the 60 musical excerpts one by one with an interval of 6 seconds in between as designed in previous studies. ${ }^{6}$ Without warning of start or end, these excerpts were presented only once through headphones, played by a computer MUSIC Jukebox software. The presentation order of the excerpts was randomized, and no more than three successive excerpts belonged to the same putative emotion. During the experiment, participants sat comfortably on the chair and the volume of the music was adjusted to a comfortable level. They were allowed to pause the presentation of music when needed. After listening to each excerpt, they were asked to choose a number corresponding to the emotional intensity they perceived, using the Likert scale (described above). During the experiment, the participants might silently name the emotion they perceived when listening to a musical excerpt, but they were instructed to rate only the intensity of emotion they perceived from that excerpt. The whole experiment lasted about 40 minutes.

\section{Statistical and data analyses}

First, the ratings of emotion intensity of the 60 musical excerpts were subjected to a principal axis analysis by a computer program, the Predictive Analytics Software Statistics, Release Version 20.0 (2013; SPSS Inc., Chicago, IL, USA). Factor loadings were rotated orthogonally using the varimax normalized method and the factors (emotion clusters) and their items (musical excerpts) were determined. Then, the number of extracted factors was determined by the scree plot as well as the parallel analysis. ${ }^{28}$ Then, the fit of factor 
Table I Information of the 60 musical excerpts: their original sources, start-stop bars, durations (seconds), playing instruments, composers, putative emotions, and acoustic features ${ }^{\mathrm{a}}$

\begin{tabular}{|c|c|c|c|c|c|c|c|}
\hline Num & Sources & Start-stop & Duration & Instruments & Composer & $\begin{array}{l}\text { Putative } \\
\text { emotion }\end{array}$ & Acoustic features \\
\hline MOI & $\begin{array}{l}\text { Nocturne in C } \\
\text { minor B. } 108\end{array}$ & Bars I-8 & 31 & \begin{tabular}{|l|} 
Piano \\
\end{tabular} & $\begin{array}{l}\text { Chopin, } \\
\text { Frédéric }\end{array}$ & Sad & $\begin{array}{l}\text { Low tempo, minor mode, low } \\
\text { sound level, legato articulation, } \\
\text { narrow pitch range, and slow } \\
\text { tone attacks }\end{array}$ \\
\hline M02 & $\begin{array}{l}\text { Minuet in G major, } \\
\text { BWV Anh. II4 }\end{array}$ & Bars $1-16$ & 23 & $\begin{array}{l}\text { Violin and } \\
\text { harpsichord }\end{array}$ & $\begin{array}{l}\text { Bach, Johann } \\
\text { Sebastian }\end{array}$ & Happy & $\begin{array}{l}\text { Medium tempo, major mode, } \\
\text { simple and consonant harmony, } \\
\text { medium-high sound level, } \\
\text { smooth and fluent rhythm }\end{array}$ \\
\hline M03 & Dream Catcher & Bars 5-12 & 24 & $\begin{array}{l}\text { Piano and } \\
\text { electronic } \\
\text { synthesizer }\end{array}$ & Rolf, Lovland & Dreamy & $\begin{array}{l}\text { Low tempo, minor mode, } \\
\text { low sound level, and legato } \\
\text { articulation }\end{array}$ \\
\hline M04 & Women of Ireland & Bars I-9 & 36 & $\begin{array}{l}\text { Flute and } \\
\text { electronic } \\
\text { synthesizer }\end{array}$ & $\begin{array}{l}\text { Joanie, } \\
\text { Madden }\end{array}$ & Mysterious & $\begin{array}{l}\text { Low tempo, sharp contrasts } \\
\text { between long and short notes, } \\
\text { legato articulation, and bright } \\
\text { timbre }\end{array}$ \\
\hline M05 & $\begin{array}{l}\text { Serenade for String } \\
\text { Orchestra Op. 48, } \\
\text { second movement }\end{array}$ & Bars I-20 & 20 & $\begin{array}{l}\text { Violin, viola, } \\
\text { and cello }\end{array}$ & $\begin{array}{l}\text { Tchaikovsky, } \\
\text { Pyotr }\end{array}$ & Fanciful & $\begin{array}{l}\text { Fast tempo, major mode, simple } \\
\text { and consonant harmony, and bright } \\
\text { timbre }\end{array}$ \\
\hline M06 & A Comme Amour & Bars 2-10 & 28 & Piano & Pagès, Philippe & Bluesy & $\begin{array}{l}\text { Low tempo, minor mode, low sound } \\
\text { level, legato articulation, and slow } \\
\text { tone attacks }\end{array}$ \\
\hline M07 & $\begin{array}{l}\text { Flight of the Bumble- } \\
\text { Bee }\end{array}$ & Bars I-38 & 28 & Accordion & $\begin{array}{l}\text { Rimsky- } \\
\text { Korsakov, } \\
\text { Nikolay }\end{array}$ & Dramatic & $\begin{array}{l}\text { Very fast tempo, rapid changes in } \\
\text { sound level, small timing variability, } \\
\text { spectral noise, and fast tone attacks }\end{array}$ \\
\hline M08 & $\begin{array}{l}\text { Piano Sonata No. } \\
\text { 8, Op. I3 }\end{array}$ & $\begin{array}{l}\text { Bars } \\
139-169\end{array}$ & 27 & Piano & $\begin{array}{l}\text { Beethoven, } \\
\text { Ludwig van }\end{array}$ & Frustrated & $\begin{array}{l}\text { Fast tempo, rapid changes in } \\
\text { sound level, sharp contrasts } \\
\text { between long and short notes, } \\
\text { and ascending pitch }\end{array}$ \\
\hline M09 & $\begin{array}{l}\text { The Nutcracker } \\
\text { (ballet) Op. 7I Act } \\
\text { 2, No. I3, Valse des } \\
\text { fleurs }\end{array}$ & Bars I-16 & 26 & Orchestra & $\begin{array}{l}\text { Tchaikovsky, } \\
\text { Pyotr }\end{array}$ & Dreamy & $\begin{array}{l}\text { Slow tempo, legato articulation, } \\
\text { medium-low sound level, small } \\
\text { sound level variability }\end{array}$ \\
\hline MIO & Hymn to Hope & Bars 4-12 & 32 & Violin & Rolf, Lovland & Longing & $\begin{array}{l}\text { Medium tempo, medium-low } \\
\text { sound level, legato articulation, } \\
\text { slow tone attacks, and soft } \\
\text { timbre }\end{array}$ \\
\hline MII & $\begin{array}{l}\text { Variations on } \\
\text { "Ah, vous dirai-je } \\
\text { maman", K. } 265\end{array}$ & Bars $1-16$ & 27 & Piano & $\begin{array}{l}\text { Mozart, } \\
\text { Wolfgang } \\
\text { Amadeus }\end{array}$ & Fanciful & $\begin{array}{l}\text { Medium tempo, major mode, } \\
\text { medium-low sound level, simple } \\
\text { rhythm }\end{array}$ \\
\hline MI2 & $\begin{array}{l}\text { The Nutcracker } \\
\text { (ballet) Op. 7la: } \\
\text { Miniature March }\end{array}$ & Bars I-16 & 27 & Orchestra & $\begin{array}{l}\text { Tchaikovsky, } \\
\text { Pyotr }\end{array}$ & Sacred & $\begin{array}{l}\text { Medium tempo, major mode, } \\
\text { staccato articulation, and fast tone } \\
\text { attacks }\end{array}$ \\
\hline MI3 & $\begin{array}{l}\text { Eine Kleine } \\
\text { Nachtmusik, K. } 525 \\
\text { second movement }\end{array}$ & Bars I-8 & 26 & $\begin{array}{l}\text { Violin, viola, } \\
\text { cello, and } \\
\text { doublebass }\end{array}$ & $\begin{array}{l}\text { Mozart, } \\
\text { Wolfgang } \\
\text { Amadeus }\end{array}$ & Graceful & $\begin{array}{l}\text { Medium tempo, major mode, } \\
\text { medium sound level, smooth and } \\
\text { fluent rhythm }\end{array}$ \\
\hline MI4 & Beer Barrel Polka & Bars 5-36 & 31 & Accordion & $\begin{array}{l}\text { Vejvoda, } \\
\text { Jaromír }\end{array}$ & Happy & $\begin{array}{l}\text { Fast tempo, staccato } \\
\text { articulation, fast tone attacks, } \\
\text { syncopation, simple and } \\
\text { consonant harmony }\end{array}$ \\
\hline MI5 & In Our Tears & Bars I-8 & 35 & $\begin{array}{l}\text { Violin and } \\
\text { piano }\end{array}$ & Rolf, Lovland & Bluesy & $\begin{array}{l}\text { Slow tempo, minor mode, } \\
\text { medium-low sound level, and legato } \\
\text { articulation }\end{array}$ \\
\hline
\end{tabular}

(Continued) 
Table I (Continued)

\begin{tabular}{|c|c|c|c|c|c|c|c|}
\hline Num & Sources & Start-stop & Duration & Instruments & Composer & $\begin{array}{l}\text { Putative } \\
\text { emotion }\end{array}$ & Acoustic features \\
\hline MI6 & $\begin{array}{l}\text { Symphony No. } 6 \\
\text { in F major, Op. } \\
68, \text { the fourth } \\
\text { movement } \\
\text { "Gewitter, Sturm" }\end{array}$ & Bars 4I-57 & 21 & Orchestra & $\begin{array}{l}\text { Beethoven, } \\
\text { Ludwig van }\end{array}$ & Agitated & $\begin{array}{l}\text { Fast tempo, large tempo } \\
\text { variability, rapid changes in } \\
\text { sound level, fast vibrato rate, } \\
\text { wide pitch range, and large } \\
\text { pitch contrasts }\end{array}$ \\
\hline MI7 & $\begin{array}{l}\text { The Sleeping Beauty } \\
\text { (ballet) Op. } 66 \text { Act II, } \\
\text { No. 17, Panorama }\end{array}$ & Bars I-18 & 38 & Orchestra & $\begin{array}{l}\text { Tchaikovsky, } \\
\text { Pyotr }\end{array}$ & Longing & $\begin{array}{l}\text { Slow tempo, major mode, medium } \\
\text { sound level, small intervals, soft } \\
\text { contrasts between long and short } \\
\text { notes }\end{array}$ \\
\hline MI8 & Evensong & Bars I-14 & 35 & $\begin{array}{l}\text { Vocal and } \\
\text { electronic } \\
\text { synthesizer }\end{array}$ & Rolf, Lovland & Mysterious & $\begin{array}{l}\text { Slow tempo, legato articulation, wide } \\
\text { pitch range, and medium fast vibrato }\end{array}$ \\
\hline MI9 & $\begin{array}{l}\text { Six Pieces, Op. } \\
\text { 5I, No. 6, Valse } \\
\text { Sentimentale }\end{array}$ & Bars I-20 & 34 & $\begin{array}{l}\text { Violin and } \\
\text { piano }\end{array}$ & $\begin{array}{l}\text { Tchaikovsky, } \\
\text { Pyotr }\end{array}$ & Sad & $\begin{array}{l}\text { Medium tempo, minor mode, wide } \\
\text { pitch range, and medium-low sound } \\
\text { level }\end{array}$ \\
\hline M20 & $\begin{array}{l}\text { Piano Sonata No. } \\
\text { 2, Op. } 3 \text { I, the third } \\
\text { movement }\end{array}$ & Bars 30-67 & 31 & Piano & $\begin{array}{l}\text { Beethoven, } \\
\text { Ludwig van }\end{array}$ & Frustrated & $\begin{array}{l}\text { Fast tempo, rapid changes in } \\
\text { sound level, wide pitch range, } \\
\text { fast tone attacks }\end{array}$ \\
\hline M2I & Loftus Jones & Bars $1-17$ & 26 & $\begin{array}{l}\text { Violin, } \\
\text { bagpipe }\end{array}$ & $\begin{array}{l}\text { Joanie, } \\
\text { Madden }\end{array}$ & Passionate & $\begin{array}{l}\text { Fast tempo, staccato } \\
\text { articulation, fast tone attacks, } \\
\text { and high pitch }\end{array}$ \\
\hline M22 & Poem & Bars 5-13 & 37 & $\begin{array}{l}\text { Violin and } \\
\text { piano }\end{array}$ & Rolf, Lovland & Graceful & $\begin{array}{l}\text { Medium-slow tempo, low sound } \\
\text { level, wide pitch range, legato } \\
\text { articulation, and slow tone attacks }\end{array}$ \\
\hline M23 & $\begin{array}{l}\text { Radetzky March, } \\
\text { Op. } 228\end{array}$ & Bars $1-20$ & 22 & Orchestra & $\begin{array}{l}\text { Strauss, } \\
\text { Johann, Sr }\end{array}$ & Happy & $\begin{array}{l}\text { Fast tempo, major mode, } \\
\text { medium-high sound level, } \\
\text { micro-structural regularity, and } \\
\text { bright timbre }\end{array}$ \\
\hline M24 & June: Barcaroole & Bars $1-12$ & 36 & Piano & $\begin{array}{l}\text { Tchaikovsky, } \\
\text { Pyotr }\end{array}$ & Bluesy & $\begin{array}{l}\text { Slow tempo, minor mode, low } \\
\text { sound level, legato articulation, } \\
\text { and rubato }\end{array}$ \\
\hline M25 & $\begin{array}{l}\text { L'Arlésienne Suite } \\
\text { No. 2, Menute }\end{array}$ & Bars $1-10$ & 21 & Flute, harp & Bizet, Georges & Dreamy & $\begin{array}{l}\text { Medium tempo, major mode, } \\
\text { ascending pitch, high pitch, soft tone } \\
\text { attacks, and legato articulation }\end{array}$ \\
\hline M26 & $\begin{array}{l}\text { The Four Seasons, } \\
\text { Spring }\end{array}$ & Bars I-10 & 22 & $\begin{array}{l}\text { Violin, viola, } \\
\text { cello, and } \\
\text { doublebass }\end{array}$ & $\begin{array}{l}\text { Vivaldi, } \\
\text { Antonio }\end{array}$ & Passionate & $\begin{array}{l}\text { Medium tempo, major mode, large } \\
\text { sound level variability, and accents } \\
\text { on tonally stable notes }\end{array}$ \\
\hline M27 & $\begin{array}{l}\text { Images, Reflects } \\
\text { dans l'eau }\end{array}$ & Bars 30-33 & 10 & Piano & $\begin{array}{l}\text { Debussy, } \\
\text { Claude }\end{array}$ & Agitated & $\begin{array}{l}\text { Fast tempo, wide pitch range, } \\
\text { disconsonant harmony, and } \\
\text { complex rhythm }\end{array}$ \\
\hline M28 & Nocturne & Bars 45-65 & 49 & $\begin{array}{l}\text { Violin and } \\
\text { electronic } \\
\text { synthesizer }\end{array}$ & Rolf, Lovland & Longing & $\begin{array}{l}\text { Medium tempo, minor mode, } \\
\text { wide pitch range, legato } \\
\text { articulation }\end{array}$ \\
\hline M29 & $\begin{array}{l}\text { The Moonlight } \\
\text { Lover }\end{array}$ & Bars 5-II & 29 & $\begin{array}{l}\text { Cello and } \\
\text { erhu }\end{array}$ & Tan, Dun & Bluesy & $\begin{array}{l}\text { Slow tempo, minor mode, } \\
\text { legato articulation, slow tone } \\
\text { attacks, and small vibrato } \\
\text { extent }\end{array}$ \\
\hline M30 & $\begin{array}{l}\text { Etudes Op. I0, } \\
\text { No. I } 2 \text { in C minor } \\
\text { "Revolutionary" }\end{array}$ & Bars $1-10$ & 16 & Piano & $\begin{array}{l}\text { Chopin, } \\
\text { Frédéric }\end{array}$ & Dramatic & $\begin{array}{l}\text { Fast tempo, wide pitch range, } \\
\text { high sound level, high note } \\
\text { density, and fast tone attacks }\end{array}$ \\
\hline M3I & $\begin{array}{l}\text { Swan Lake (ballet), } \\
\text { Op. } 20 \text { Act I Scene }\end{array}$ & Bars I-9 & 28 & $\begin{array}{l}\text { Oboe, harp, } \\
\text { violin }\end{array}$ & $\begin{array}{l}\text { Tchaikovsky, } \\
\text { Pyotr }\end{array}$ & Dreamy & $\begin{array}{l}\text { Slow tempo, minor mode, } \\
\text { legato articulation, and soft } \\
\text { timbre }\end{array}$ \\
\hline M32 & $\begin{array}{l}\text { Prelude of the } \\
\text { Spring Festival }\end{array}$ & Bars I-34 & 29 & Orchestra & Li, Huanzhi & Passionate & $\begin{array}{l}\text { Fast tempo, high sound level, } \\
\text { high pitch, bright timbre, and } \\
\text { accents on tonally stable notes }\end{array}$ \\
\hline
\end{tabular}

(Continued) 
Table I (Continued)

\begin{tabular}{|c|c|c|c|c|c|c|c|}
\hline Num & Sources & Start-stop & Duration & Instruments & Composer & $\begin{array}{l}\text { Putative } \\
\text { emotion }\end{array}$ & Acoustic features \\
\hline M33 & The Rite of Spring & Bars 9-24 & 15 & Orchestra & $\begin{array}{l}\text { Stravinsky, } \\
\text { Igor }\end{array}$ & Agitated & $\begin{array}{l}\text { Fast tempo, dissonance, } \\
\text { staccato articulation, and large } \\
\text { pitch contrasts }\end{array}$ \\
\hline M34 & The Pledge of Love & Bars I-I5 & 43 & $\begin{array}{l}\text { Piano and } \\
\text { electronic } \\
\text { synthesizer }\end{array}$ & Pagès, Philippe & Graceful & $\begin{array}{l}\text { Medium tempo, small sound level } \\
\text { variability, soft contrasts between } \\
\text { long and short notes, and micro- } \\
\text { structural regularity }\end{array}$ \\
\hline M35 & $\begin{array}{l}\text { Piano Sonata No. } \\
\text { I4, Op. 27, No. } 2 \text {, } \\
\text { Adagio Sostenuto }\end{array}$ & Bars I-4 & 23 & Piano & $\begin{array}{l}\text { Beethoven, } \\
\text { Ludwig van }\end{array}$ & Dreamy & $\begin{array}{l}\text { Slow tempo, minor mode, low } \\
\text { sound level, and rubato }\end{array}$ \\
\hline M36 & $\begin{array}{l}\text { The Carnival of } \\
\text { the Animals, The } \\
\text { Aquarium }\end{array}$ & Bars $1-12$ & 39 & $\begin{array}{l}\text { Piano and } \\
\text { violin }\end{array}$ & $\begin{array}{l}\text { Saint-Saëns, } \\
\text { Camille }\end{array}$ & Mysterious & $\begin{array}{l}\text { Medium tempo, high note density, } \\
\text { high pitch, and medium-low sound } \\
\text { level }\end{array}$ \\
\hline M37 & $\begin{array}{l}\text { Swan Lake } \\
\text { (ballet), Op. } 20 \\
\text { Act } 2 \text {, Danses des } \\
\text { cygenes }\end{array}$ & Bars I-9 & 23 & $\begin{array}{l}\text { Tuba and } \\
\text { violin }\end{array}$ & $\begin{array}{l}\text { Tchaikovsky, } \\
\text { Pyotr }\end{array}$ & Happy & $\begin{array}{l}\text { Fast tempo, minor mode, } \\
\text { staccato articulation, and bright } \\
\text { timbre }\end{array}$ \\
\hline M38 & River of Sorrow & Bar I & 60 & $\begin{array}{l}\text { Orchestra and } \\
\text { cello }\end{array}$ & Huang, Haihuai & Sad & $\begin{array}{l}\text { Medium-slow tempo, large timing } \\
\text { variability, falling intonation, small } \\
\text { vibrato extent, and ritardando }\end{array}$ \\
\hline M39 & $\begin{array}{l}\text { Symphony No. } 6 \text { in } \\
\text { F major, Op. } 68\end{array}$ & Bars |7-4| & 32 & Orchestra & $\begin{array}{l}\text { Beethoven, } \\
\text { Ludwig van }\end{array}$ & Frustrated & $\begin{array}{l}\text { Fast tempo, high sound level, } \\
\text { wide pitch range, and high note } \\
\text { density }\end{array}$ \\
\hline M40 & $\begin{array}{l}\text { Piano Sonata No. } 2 \\
\text { in d minor, Op. } 3 \text { I }\end{array}$ & Bars $|-2|$ & 17 & Piano & $\begin{array}{l}\text { Beethoven, } \\
\text { Ludwig van }\end{array}$ & Fanciful & $\begin{array}{l}\text { Fast tempo, smooth and fluent } \\
\text { rhythm, small timing variability, } \\
\text { and medium sound level }\end{array}$ \\
\hline M4I & $\begin{array}{l}\text { Symphony No. } 3 \\
\text { in Eb major Op. } 55 \\
\text { "eroicae" }\end{array}$ & $\begin{array}{l}\text { Bars } \\
382-398\end{array}$ & 47 & Orchestra & $\begin{array}{l}\text { Beethoven, } \\
\text { Ludwig van }\end{array}$ & Sacred & $\begin{array}{l}\text { Medium tempo, accents on } \\
\text { tonally stable note, firm rhythm, } \\
\text { and narrow pitch range }\end{array}$ \\
\hline M42 & $\begin{array}{l}\text { Swan Lake (ballet), } \\
\text { Op. } 20 \text { Act 3, No. } \\
\text { 23, Mazurka }\end{array}$ & Bars I-24 & 34 & Orchestra & $\begin{array}{l}\text { Tchaikovsky, } \\
\text { Pyotr }\end{array}$ & Passionate & $\begin{array}{l}\text { Fast tempo, high sound level, } \\
\text { small sound level variability, and } \\
\text { high pitch }\end{array}$ \\
\hline M43 & Adagio & Bars 2-6 & 17 & $\begin{array}{l}\text { Violin, } \\
\text { cello, and } \\
\text { electronic } \\
\text { synthesizer }\end{array}$ & Rolf, Lovland & Graceful & $\begin{array}{l}\text { Slow tempo, high sound level, } \\
\text { legato articulation, soft timbre, } \\
\text { and slow tone attacks }\end{array}$ \\
\hline M44 & $\begin{array}{l}\text { The Carnival of } \\
\text { the Animals, The } \\
\text { Swan }\end{array}$ & Bars I-5 & 33 & $\begin{array}{l}\text { Cello and } \\
\text { piano }\end{array}$ & $\begin{array}{l}\text { Saint-Saëns, } \\
\text { Camille }\end{array}$ & Bluesy & $\begin{array}{l}\text { Slow tempo, falling intonation, } \\
\text { medium-low sound level, legato } \\
\text { articulation, and soft timbre }\end{array}$ \\
\hline M45 & $\begin{array}{l}\text { Peer Gynt Suite } \\
\text { No. 2, Op. } 55 \text {, } \\
\text { "Solveigs sang" }\end{array}$ & Bars 8-17 & 36 & Flute, harp & $\begin{array}{l}\text { Grieg, } \\
\text { Edvard }\end{array}$ & Dreamy & $\begin{array}{l}\text { Slow tempo, minor mode, } \\
\text { legato articulation, slow tone } \\
\text { attacks, and soft timbre }\end{array}$ \\
\hline M46 & $\begin{array}{l}\text { Five Orchestra } \\
\text { Pieces, Op. 16, } \\
\text { No. } 5\end{array}$ & Bars $1-17$ & 24 & Orchestra & $\begin{array}{l}\text { Schoenberg, } \\
\text { Arnold }\end{array}$ & Agitated & $\begin{array}{l}\text { Large tempo variability, } \\
\text { disconsonate harmony, } \\
\text { atonality, sharp contrasts } \\
\text { between long and short notes }\end{array}$ \\
\hline M47 & $\begin{array}{l}\text { Symphony No. } 4 \text { in } f \\
\text { minor Op. } 36 \text { Finale }\end{array}$ & Bars 17-59 & 62 & Orchestra & $\begin{array}{l}\text { Tchaikovsky, } \\
\text { Pyotr }\end{array}$ & Dramatic & $\begin{array}{l}\text { Fast tempo, high sound level, high } \\
\text { note density, accents on tonally } \\
\text { stable note, and firm rhythm }\end{array}$ \\
\hline M48 & Homesick Song & Bars I-8 & 24 & $\begin{array}{l}\text { Violin and } \\
\text { piano }\end{array}$ & Ma, Sicong & Bluesy & $\begin{array}{l}\text { Slow tempo, minor mode, } \\
\text { legato articulation, and narrow } \\
\text { pitch range }\end{array}$ \\
\hline M49 & $\begin{array}{l}\text { Symphony No. } 6 \text { in b } \\
\text { minor Op. 74, fourth } \\
\text { movement }\end{array}$ & Bars 19-25 & 25 & Orchestra & $\begin{array}{l}\text { Tchaikovsky, } \\
\text { Pyotr }\end{array}$ & Frustrated & $\begin{array}{l}\text { Slow tempo, minor mode, large } \\
\text { timing variability, and rubato }\end{array}$ \\
\hline
\end{tabular}


Table I (Continued)

\begin{tabular}{|c|c|c|c|c|c|c|c|}
\hline Num & Sources & Start-stop & Duration & Instruments & Composer & $\begin{array}{l}\text { Putative } \\
\text { emotion }\end{array}$ & Acoustic features \\
\hline M50 & Poem & Bars 69-75 & 31 & $\begin{array}{l}\text { Violin and } \\
\text { electronic } \\
\text { synthesizer }\end{array}$ & Rolf, Lovland & Sad & $\begin{array}{l}\text { Medium-slow tempo, minor } \\
\text { mode, legato articulation, and } \\
\text { soft timbre }\end{array}$ \\
\hline M5I & $\begin{array}{l}\text { The Girl With The } \\
\text { Flaxen Hair }\end{array}$ & Bars 7-17 & 41 & $\begin{array}{l}\text { Violin and } \\
\text { piano }\end{array}$ & $\begin{array}{l}\text { Debussy, } \\
\text { Claude }\end{array}$ & Longing & $\begin{array}{l}\text { Slow tempo, high pitch, medium-low } \\
\text { sound level, and legato articulation }\end{array}$ \\
\hline M52 & Double Concertos & Bars $1-10$ & 29 & $\begin{array}{l}\text { Violin and } \\
\text { viola }\end{array}$ & $\begin{array}{l}\text { Bach, Johann } \\
\text { Sebastian }\end{array}$ & Sacred & $\begin{array}{l}\text { Medium tempo, firm rhythm, } \\
\text { minor mode, and micro- } \\
\text { structural regularity }\end{array}$ \\
\hline M53 & Children of the River & Bars I-9 & 32 & $\begin{array}{l}\text { Bamboo flute } \\
\text { and electronic } \\
\text { synthesizer }\end{array}$ & Rolf, Lovland & Mysterious & $\begin{array}{l}\text { Medium tempo, large timing } \\
\text { variability, and small vibrato extent }\end{array}$ \\
\hline M54 & Couleur Tendresse & Bars I-9 & 30 & Piano & $\begin{array}{l}\text { Pagès, } \\
\text { Philippe }\end{array}$ & Graceful & $\begin{array}{l}\text { medium tempo, medium-low } \\
\text { sound level, slow tone attacks, } \\
\text { and soft timbre }\end{array}$ \\
\hline M55 & $\begin{array}{l}\text { Swan Lake (ballet), } \\
\text { Op. } 20 \text { Act 3, Danse } \\
\text { Napolitaine }\end{array}$ & Bars 5-15 & 25 & $\begin{array}{l}\text { Trumpet and } \\
\text { orchestra }\end{array}$ & $\begin{array}{l}\text { Tchaikovsky, } \\
\text { Pyotr }\end{array}$ & Happy & $\begin{array}{l}\text { Medium tempo, staccato articulation, } \\
\text { syncopation, and bright timbre }\end{array}$ \\
\hline M56 & Dance of Sarasvati & Bars I-16 & 38 & $\begin{array}{l}\text { Electronic } \\
\text { synthesizer }\end{array}$ & $\begin{array}{l}\text { Masanori, } \\
\text { Takahashi }\end{array}$ & Mysterious & $\begin{array}{l}\text { Medium tempo, medium-low sound } \\
\text { level, and large pitch range }\end{array}$ \\
\hline M57 & Serenade to Spring & Bars 4-28 & 49 & $\begin{array}{l}\text { Violin and } \\
\text { piano }\end{array}$ & Rolf, Lovland & Longing & $\begin{array}{l}\text { Medium tempo, medium-low } \\
\text { sound level, narrow pitch range, } \\
\text { simple rhythm, and bright } \\
\text { timbre }\end{array}$ \\
\hline M58 & $\begin{array}{l}\text { Swan Lake (ballet), } \\
\text { Op. } 20 \text { Act 3, } \\
\text { No. 2I, Danse } \\
\text { Espagnole }\end{array}$ & Bars $1-18$ & 23 & $\begin{array}{l}\text { Flute and } \\
\text { orchestra }\end{array}$ & $\begin{array}{l}\text { Tchaikovsky, } \\
\text { Pyotr }\end{array}$ & Dramatic & $\begin{array}{l}\text { Fast tempo, large sound level } \\
\text { variability, staccato articulation, } \\
\text { syncopation, and bright timbre }\end{array}$ \\
\hline M59 & $\begin{array}{l}\text { Divertimento in } \\
\text { D major, K. 334, } \\
\text { third movement, } \\
\text { Menuetto }\end{array}$ & Bars $1-16$ & 26 & $\begin{array}{l}\text { Violin and } \\
\text { electronic } \\
\text { synthesizer }\end{array}$ & $\begin{array}{l}\text { Mozart, } \\
\text { Wolfgang } \\
\text { Amadeus }\end{array}$ & Fanciful & $\begin{array}{l}\text { Medium tempo, major mode, } \\
\text { simple rhythm, soft contrasts } \\
\text { between long and short notes }\end{array}$ \\
\hline M60 & The Blue Danube & Bars 2-II & 33 & Orchestra & $\begin{array}{l}\text { Strauss, } \\
\text { Johann, Jr }\end{array}$ & Sacred & $\begin{array}{l}\text { Slow tempo, minor mode, large } \\
\text { timing variability, rubato, and } \\
\text { slow tone attacks }\end{array}$ \\
\hline
\end{tabular}

Note: ${ }^{\text {a The }} 38$ excerpts finally retained are given in bold for clarity.

model was evaluated by AMOS, ${ }^{29}$ a CFA for the structural equation modeling on the same participant sample to evaluate the preliminary test of the model fit proposed by EFA. We conducted both EFA and CFA on the same sample for a cross-validation procedure as conducted previously. ${ }^{30-32}$

The factors whose items conveyed significantly different emotions were subjected to a second round of principal axis analysis to obtain sub-factors (facets). Again, the facet loadings were rotated orthogonally via the varimax normalized method. The number of extracted facets in the following analysis was determined by the scree plot and eigenvalues. Items which were loaded less heavily (below 0.4 ) on the target facet or cross-loaded heavily (above 0.4 ) were removed. As performed in the first-level factor, the facet model was evaluated via CFA and the goodness of fit of final model was tested. Then, the internal reliabilities (Cronbach's $\alpha$ values) of each facet were analyzed. Finally, we applied two-way ANOVA (Gender $\times$ Emotion) to detect the perceived emotional intensity differences between gender and emotion. Once significance was detected, the post hoc test of the least significant difference method was conducted to detect the effect of gender or emotion. A $P$-value $<0.05$ was considered as significant.

\section{Results}

The principal axis factor analysis revealed nine eigenvalues higher than 1.0, which were 20.42, 4.55, 2.58, 1.62, 1.32, $1.26,1.16,1.06$, and 1.03, respectively. The scree plot (Figure 2) indicated approximately four factors, which were the same as parallel analysis indicated. The first three, four, five, 


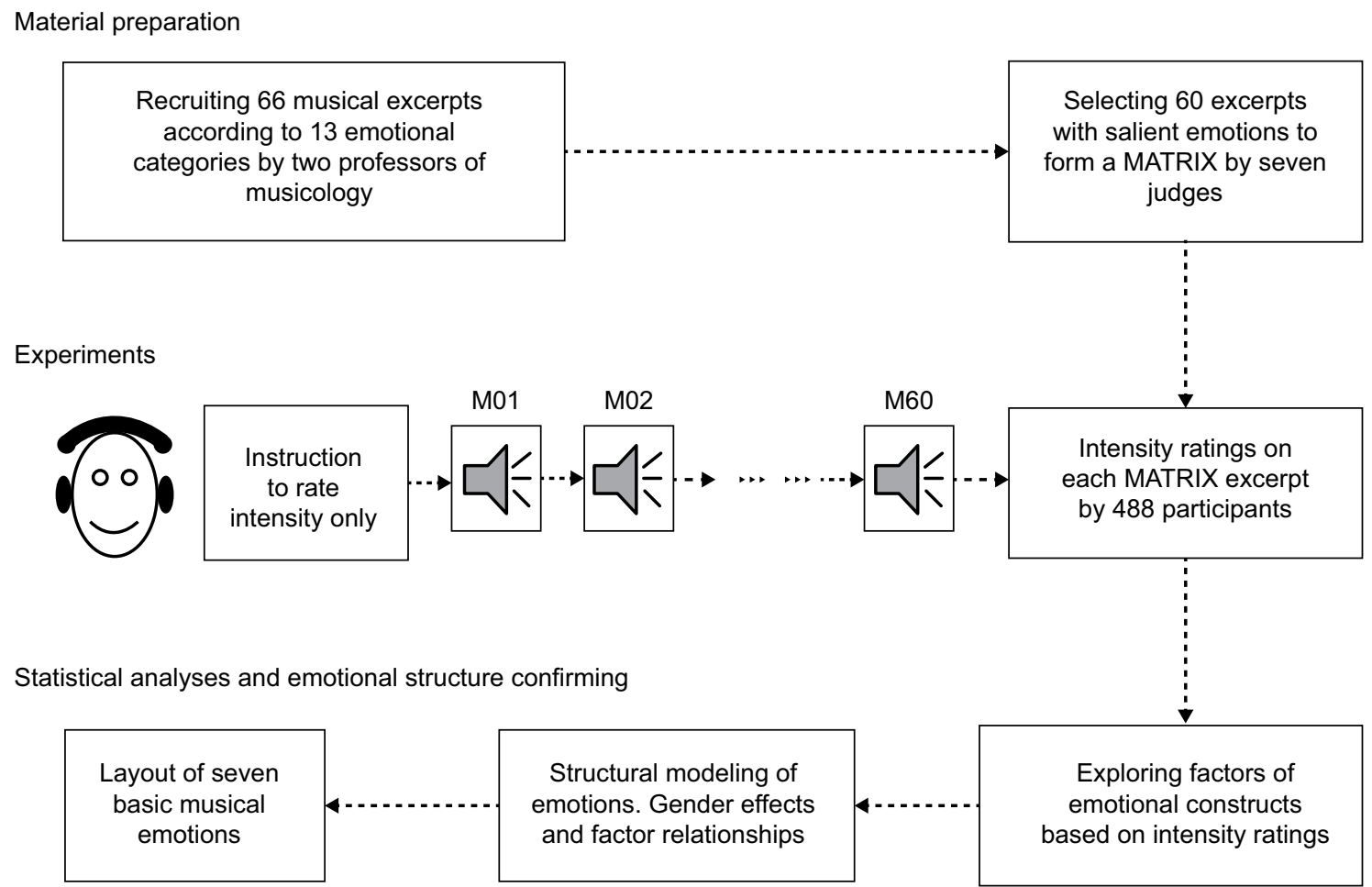

Figure I Flowchart of the experimental design.

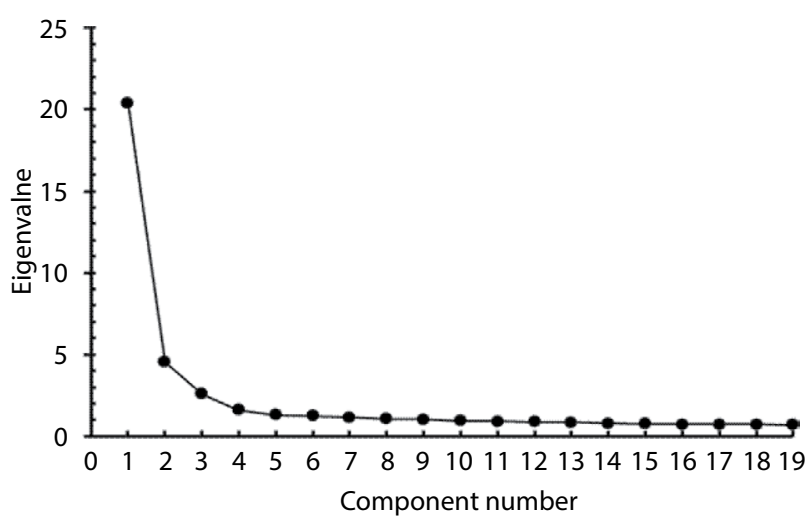

Figure 2 Scree plot from the exploratory factor analysis of the intensity ratings on 60 musical excerpts in 488 participants.

and six factors accounted for $45.92 \%, 48.61 \%, 50.81 \%$, and $52.91 \%$ of the total variance, respectively. Hence, we established three-, four-, five-, and six-factor modeling, and then evaluated the model fit parameters; the results indicated that three-factor model was the most suitable one (Table 2). These three factors contained 26, 17, and 17 music excerpts, with Cronbach's $\alpha$ values of $0.95,0.92$, and 0.91 , respectively.

For factor 1 , the ratings of intensity of emotion on the 26 excerpts were analyzed again by principal axis factor analysis, and the varimax normalized method rotation, for the second-order layout. Three facets with eigenvalues of $11.02,1.37$, and 1.09, respectively, came out, accounting for $51.88 \%$ of the total variance. With the loading criteria, three excerpts (M13, M22, and M34) were removed from the first facet and three (M18, M38, and M56) from the third. Out of the remaining ones, two excerpts (M15 and M25) within the first facet and two (M06 and M36) within the second facet which displayed distinct emotion from the others were deleted. Eventually, the first facet consisting of seven excerpts, mostly in minor mode, played by instruments such as piano, oboe, flute, and violin, expressed with slow tempo, little variability of sound level, soft change between long and short notes, legato melody with long phrases, and soft timbre, was named Tenderness. The second facet with five excerpts possessing moderate tempo, simple and consonant harmony, high pitch range, firm rhythm, and fast tone attack was named Happiness. Consistent with the first facet, the melodic instruments used in the excerpts were violin and piano. The third facet with four excerpts featured with slow tempo, low note density, minor mode, little pitch variability, consonant harmony, descending melodic lines, and soft timbre, was played by flute, violin, and cello, and was called Sadness. The internal consistencies of these three facets were $0.85,0.80$, and 0.72 , respectively (Table 3 ). 
Table 2 Model fit parameters based on the first-order analyses in 488 participants

\begin{tabular}{|l|l|l|l|l|l|l|l|}
\hline Factor model & $\chi^{2} / d f$ & $\begin{array}{l}\text { Goodness of } \\
\text { fit index }\end{array}$ & $\begin{array}{l}\text { Adjusted } \\
\text { goodness of } \\
\text { fit index }\end{array}$ & $\begin{array}{l}\text { Tucker- } \\
\text { Lewis } \\
\text { index }\end{array}$ & $\begin{array}{l}\text { Comparative } \\
\text { fit index }\end{array}$ & $\begin{array}{l}\text { Root mean } \\
\text { square error of } \\
\text { approximation }\end{array}$ & $\begin{array}{l}\text { Standardized } \\
\text { root square } \\
\text { residual }\end{array}$ \\
\hline Three-factor model & 2.78 & 0.72 & 0.70 & 0.79 & 0.80 & 0.060 & 0.070 \\
Four-factor model & 2.77 & 0.72 & 0.70 & 0.79 & 0.80 & 0.060 & 0.073 \\
Five-factor model & 2.71 & 0.73 & 0.71 & 0.80 & 0.80 & 0.059 & 0.070 \\
Six-factor model & 2.57 & 0.75 & 0.73 & 0.81 & 0.82 & 0.057 & 0.066 \\
\hline
\end{tabular}

Abbreviation: $d f$, degrees of freedom.

Table 3 The internal consistencies of seven music domains (second-order level) in all participants $(n=488)$ and the intensity ratings (mean $\pm S D)$ of each domain in female $(n=274)$ and male $(n=2 / 4)$ participants

\begin{tabular}{|c|c|c|c|c|}
\hline \multirow[t]{2}{*}{ Emotion } & \multirow[t]{2}{*}{ Music excerpts } & \multirow[t]{2}{*}{ Internal $\alpha$} & \multicolumn{2}{|c|}{ Intensity rating } \\
\hline & & & Female & Male \\
\hline Tenderness & M0I, M03, MIO, M24, M3I, M43, M45 & 0.85 & $4.30 \pm 1.26$ & $4.22 \pm 1.34$ \\
\hline Happiness & M02, MII, M37, M57, M59 & 0.80 & $5.02 \pm 1.25$ & $4.97 \pm 1.30$ \\
\hline Sadness & M04, M28, M29, M50 & 0.72 & $5.07 \pm 1.20$ & $4.92 \pm 1.27$ \\
\hline Anger & M08, MI6, M20, M33, M39, M4I, M42, M52, M58 & 0.87 & $5.38 \pm 1.13$ & $5.33 \pm 1.32$ \\
\hline Passion & MI4, M2I, M23, M32 & 0.80 & $6.09 \pm 1.15$ & $5.97 \pm 1.21$ \\
\hline Depression & M35, M44, M46, M48, M54, M60 & 0.81 & $4.30 \pm 1.29$ & $4.23 \pm 1.20$ \\
\hline Anxiousness & M27, M30, M40 & 0.69 & $4.6 I \pm I .23$ & $4.48 \pm 1.20$ \\
\hline
\end{tabular}

For factor 2, the principal axis factor analysis to run for the second-order layout revealed two facets with eigenvalues of 7.57 and 1.23 , which accounted for $51.75 \%$ of the total variance. According to the loading criteria, two excerpts (M12 and M47) and one excerpt (M26) were removed from the fourth and fifth facets, respectively. Moreover, one excerpt (M07) was excluded from the fifth facet since it expressed distinguished emotion from the others. Finally, the fourth facet including nine musical excerpts with characteristics of more tonal modulations, faster tempo, higher note density, more dissonant and complex harmony, faster tone attacks, and stronger intensity fluctuations was named Anger. The fifth facet with four excerpts was named Passion, accompanying with features of faster tempo, more staccato articulations, higher note density, and stronger intensity fluctuations. In most excerpts with these two emotions, percussion instruments were used frequently to establish an intense atmosphere. The internal consistency was 0.87 for Anger and 0.80 for Passion (Table 3).

For factor 3, two facets with eigenvalues of 7.05 and 1.05 emerged after the second-order oriented principal axis factor analysis, which accounted for $47.65 \%$ of the total variance. Two items (M09 and M49) of the sixth facet and three items (M05, M55, and M51) of the seventh facet were removed due to their cross-loadings $>0.40$. One item (M53) and other two (M19 and M40) were removed from the sixth and seventh facets, respectively, due to their less congruence in emotion with the others. The sixth facet with six items was named Depression, with slow tempo, low note density, little intensity fluctuations, and falling melodic lines, and in minor mode. Most excerpts were played with instruments in soft timbre, such as violin, cello, and piano, and French horn which was grouped together in orchestration. The seventh facet with three items exhibiting more tonal modulations, faster tempo, higher note density, stronger intensity fluctuations, and wider pitch range was named Anxiousness. All excerpts were played with piano. The internal consistencies were 0.81 and 0.69 for Depression and Anxiousness, respectively (Table 3 ).

The remaining 38 music excerpts under seven facets constructed a model with satisfactory construct validities as indicated by parameters such as the $\chi^{2} / d f$ of 2.48 , goodness of fit index 0.84 , adjusted goodness of fit index 0.82 , Tucker-Lewis index 0.87 , comparative fit index 0.88 , root mean square error of approximation 0.055 , and standardized root square residual 0.062 . There was no gender effect on intensity ratings (Gender effect: $F(1,487)=1.16, P=0.28$, mean square effect $[\mathrm{MSE}]=7.54$; Emotion $\times$ Gender interaction: $F(6,2,922)=0.26, P=0.96, \mathrm{MSE}=0.17)$, although there was an emotional effect on intensity ratings (Emotion effect: $F(6,2,922)=293.32, P<0.001, \mathrm{MSE}=195.99)$, as also found in Table 3 . The intensity of various emotions compared in 
pairs showed any two of these emotions were significantly different, except Sadness and Happiness, and Tenderness and Depression. The intercorrelations (ranging from 0.44 to 0.75 ) between any two emotions were significant (Table 4).

\section{Discussion}

We found seven emotions using both EFA and CFA on the intensity rating of musical excerpts, that is, Tenderness, Happiness, Sadness, Anger, Passion, Depression, and Anxiousness, which partly supported our hypothesis. The parameters of fitting model were satisfactory, indicating the seven music emotion model displayed nice construct validity; the internal $\alpha$ of each emotional domain was also satisfactory, indicating each possessed good internal consistency. Unfortunately, we did not find any gender difference on the intensity ratings on the musical emotions perceived by participants.

Five emotions, that is, Happiness, Sadness, Tenderness, Anger, and Anxiousness, found in our study were well acknowledged as the basic ones in music field. ${ }^{19,33,34}$ They were always among the top ten answers when individuals were asked which emotion might be expressed by music, ${ }^{17,35}$ and they even could be communicated with a high accuracy which was comparable to the facial and vocal emotions..$^{21,34}$ Our results were in line with that these emotions were easily recognized by various populations with a high accuracy. ${ }^{19}$ Happiness and Sadness were the most identifiable ones in music, ${ }^{34}$ for they were relatively effortless to express due to their fairly consistent but different features when referring to their mode and tempo. ${ }^{36}$ Tenderness was not simply a general positive emotion, ${ }^{37}$ but was obvious in both music performance and vocal expression, and it was highly recognized in previous studies. ${ }^{21}$ Both Anger and Anxiousness had negative valence and high arousal, but they were different in various aspects. ${ }^{21,34,38}$ For instance, Anxiousness was often categorized into "fear family", and was an emotion oriented to the future; this feature was different from Anger which was oriented to the present. ${ }^{21}$ Indeed, both fear and anger are associated with acoustic features such as fast tempo, increased sound level variability, and high pitch. However, anger is conveyed through higher sound level, higher-frequency energy, and more pitch variability than those of fear. ${ }^{34}$ Furthermore, these two musical emotions function differently; angry stimuli exposure could induce the experience of anger and thus modulated individuals' own emotion, but anxious listeners hardly immersed themselves into anxiety. ${ }^{38,39}$

In our study, passion facet first emerged together with Anger, which might be due to that most excerpts with these two emotions were played with not only rhythmic instruments but also colorful instruments such as in percussion. It was similar to Hevner's adjective circle, where agitated and passionate were in the same cluster. ${ }^{13}$ This affective cluster was characterized by fast tempo, low pitch, firm rhythm, complex harmony, and descending melody. ${ }^{40}$ However, in our study, we found our passionate musical excerpts were accompanied with features of uniform modality and consonant harmony, while musical excerpts in Anger showed increased modal interchanges and dissonant harmony. Therefore, Passion was presented as an independent emotion, as it was characterized to one of the five emotions in a previous report ${ }^{41}$ and it was supported that passion together with anger were one of the best labels describing musical emotion. ${ }^{42}$ In addition, in music information retrieval and musical emotion recognition research, passion emerged with the sound and music features such as timbral texture, rhythmic content, and pitch content through various algorithms. ${ }^{15,42,43}$

In the current study, we found that Depression and Sadness were independent emotions rather than belonging to the "sad family". In Hevner's adjective circle, sadness and depression together with other sad-colored adjectives were classified into one domain that reflected sadness-loaded emotion, ${ }^{13}$ which was confirmed in other similar lexical studies. ${ }^{14,16,18,44}$ However, when the participants recognize broad emotion family with a high agreement, they might also perceive nuance within the big emotion family, though with a low agreement. ${ }^{26}$ For instance, Brown found that the agreement of sorting music into six broad emotions was high, while the agreement of sorting nuances or variants of this quality was low. ${ }^{45}$ Actually, sadness-like emotion possesses many variants such as

Table 4 Inter-relationships between seven musical emotions in 488 participants

\begin{tabular}{|l|l|l|l|l|l|l|}
\hline & Tenderness & Happiness & Sadness & Anger & Passion & Depression \\
\hline Happiness & 0.72 & & & & & \\
Sadness & 0.62 & 0.62 & 0.47 & 0.48 & & \\
Anger & 0.38 & 0.66 & 0.53 & & & \\
Passion & 0.45 & 0.65 & 0.52 & 0.64 & & \\
Depression & 0.75 & 0.51 & 0.47 & 0.68 & 0.44 & \\
Anxiousness & 0.54 & & 0.47 & \\
\hline
\end{tabular}

Note: All correlations were significant at $P<0.001$. 
passiveness and grief, ${ }^{33}$ and people could perceive various sadness-like emotions when listening to sad music..44,46 In our study, based on the intensity perceived only, irrespective of the semantic considerations of emotion, we separated the two classes of sad-like music. Our results were supported by a clinical consideration that depression was a severe form or medicalization label of sadness. ${ }^{47}$ Moreover, in music, it has been consistently found that sad music induces negative, positive, and mixed emotions, ${ }^{44,48,49}$ and music-evoked sadness is composed of grief, melancholia, and sweet sorrow. ${ }^{50}$ Although many factors such as personality, memorable experience, preference, and musical expectancy might account for such intriguing results, ${ }^{51}$ one possible interpretation is that researchers did not distinguish the subtypes of sadness-like music, since they also conveyed positive emotions such as romance and some blithe beyond sadness. ${ }^{44}$

Intercorrelations between emotions were at medium to high levels in our study. On one hand, it might be due to the intensity rating method, since previous results showed that individuals' background had no effect on emotion ratings. ${ }^{52}$ On the other hand, musical emotions were often experienced in a blended manner, rather than being fully separated. ${ }^{18}$ Nonetheless, these intercorrelations reflected the complexity of emotion perception in musical context, for example, a piece of music could express both joy and surprise. ${ }^{52}$ Moreover, mixed emotions expressed in music might produce nuanced feelings, causing low satisfactory agreement on them among individuals. ${ }^{53}$

The present study has also suffered from some design flaws. Firstly, most musical excerpts were chosen from the classical genre and the number of each musical excerpt was small. Different genres might show different musical emotions. Further studies might be expanded to various genres of music. Moreover, our coauthors acted as judges to filter the musical excerpts, which might be relatively subjective. A more neutral group of specialists rather than coauthors might guarantee stronger quality control of the MATRIX. Secondly, our participants were young adults and less musicologically trained, which might lead to the nonsignificant gender difference as suggested by previous results. ${ }^{54}$ Whether our results could be extended into general or music-specialized population needs to be confirmed. Thirdly, we focused on the intensity only, and whether other methods such as emotion labeling (through categorical or nominal approach) would yield differently remains to be determined. Meanwhile, in order to provide as many musical excerpts with salient emotion as possible, we neither controlled the instruments used, nor the duration or the volume of each musical excerpt strictly, which might affect the intensity ratings of our partici- pants. Future research might use artificial musical pieces or standardize the musical structure and duration to control the musical emotion perception. Fourthly, many cross-cultural studies have shown that Westerners prefer emotions of higher arousal than the Easterners do.$^{55}$ Our participants were all Chinese; therefore, whether there was a cultural influence on the intensity ratings of our participants remains to be seen.

\section{Conclusion}

Our study was free from the influence of lexicons or musicological background, which might provide a new way to delineate basic musical emotions. Indeed, through both EFA and CFA on the intensity ratings only, we have delineated seven musical emotions: Tenderness, Happiness, Sadness, Anger, Passion, Depression, and Anxiousness.

\section{Ethics statement}

The research conformed to the Helsinki Declaration concerning human rights and informed consent, and followed correct procedures concerning treatment of humans in research.

\section{Acknowledgments}

The authors would like to thank Shanhui Shen, Yaoxi Chen, Hongying Fan, Jiawei Wang, Guorong Ma, Qisha Zhu, Bingren Zhang, and Xu Shao for their assistance during the experiment.

\section{Author contributions}

WW conceived the study and participated in the design and coordination of the study. CS, MW, LS, and CW conducted the tests. All authors contributed to data analysis, drafting and revising the article, gave final approval of the version to be published, and agree to be accountable for all aspects of the work.

\section{Disclosure}

The study was supported by a grant from the Natural Science Foundation of China (No. 81771475) to the corresponding author Dr Wei Wang. Dr Javier Cabanyes-Truffino is currently working in the Faculty of Psychology, University of Navarre, Pamplona, Spain. The authors report no other conflicts of interest in this work.

\section{References}

1. Jain R, Bagdare S. Music and consumption experience: a review. Int J Retail Distrib Manag. 2011;39(4):289-302.

2. Saarikallio S, Nieminen S, Brattico E. Affective reactions to musical stimuli reflect emotional use of music in everyday life. Music Sci. 2013;17(1):27-39.

3. Finlay KA, Anil K. Passing the time when in pain: investigating the role of musical valence. Psychomusicology. 2016;26(1):56-66. 
4. Erkkilä J, Gold C, Fachner J, Ala-Ruona E, Punkanen M, Vanhala $M$. The effect of improvisational music therapy on the treatment of depression: protocol for a randomised controlled trial. BMC Psychiatry. 2008;8(1):50.

5. Peretz I. Happy, sad, scary and peaceful musical excerpts for research on emotions. Cogn Emot. 2008;22(4):720-752.

6. Song Y, Dixon S, Pearce MT, Halpern AR. Perceived and induced emotion responses to popular music: categorical and dimensional models. Music Percept. 2016;33(4):472-492.

7. Russell JA. A circumplex model of affect. J Pers Soc Psychol. 1980;39(6):1161-1178.

8. Watson D, Tellegen A. Toward a consensual structure of mood. Psychol Bull. 1985;98(2):219-235.

9. Watson D, Wiese D, Vaidya J, Tellegen A. The two general activation systems of affect: structural findings, evolutionary considerations, and psychobiological evidence. J Pers Soc Psychol. 1999;76(5):820-838.

10. Bradley MM, Greenwald MK, Petry MC, Lang PJ. Remembering pictures: pleasure and arousal in memory. J Exp Psychol Learn Mem Cogn. 1992;18(2):379-390.

11. Rubin DC, Talarico JM. A comparison of dimensional models of emotion: evidence from emotions, prototypical events, autobiographical memories, and words. Memory. 2009;17(8):802-808.

12. Khalfa S, Isabelle P, Jean-Pierre B, Manon R. Event-related skin conductance responses to musical emotions in humans. Neurosci Lett. 2002;328(2):145-149.

13. Hevner K. Experimental studies of the elements of expression in music. Am J Psychol. 1936;48(2):246-268.

14. Farnsworth PR. A study of the Hevner adjective list. JAesthet Art Critici. 1954;13(1):97-103.

15. Li T, Ogihara M. Detecting emotion in music. Paper presented at: International Symposium/Conference on Music Information Retrieval; October 27-30; 2003; Baltimore, MD, USA.

16. Schubert E. Update of the Hevner adjective checklist. Percept Mot Skills. 2003;96(3 Pt 2):1117-1122.

17. Juslin PN, Laukka P. Expression, perception, and induction of musical emotions: a review and a questionnaire study of everyday listening. J New Music Res. 2004;33(3):217-238.

18. Zentner M, Grandjean D, Scherer KR. Emotions evoked by the sound of music: characterization, classification, and measurement. Emotion. 2008;8(4):494-521.

19. Eerola T, Vuoskoski JK. A review of music and emotion studies: approaches, emotion models, and stimuli. Music Percept. 2013;30(3):307-340.

20. Hupka RB, Lenton AP, Hutchison KA. Universal development of emotion categories in natural language. J Pers Soc Psychol. 1999;77(2):247-278.

21. Juslin PN, Laukka P. Communication of emotions in vocal expression and music performance: different channels, same code? Psychol Bull. 2003;129(5):770-814

22. Rosellini AJ, Brown TA. The NEO Five-Factor Inventory: latent structure and relationships with dimensions of anxiety and depressive disorders in a large clinical sample. Assessment. 2011;18(1):27-38.

23. Clarke DM, Kissane DW, Trauer T, Smith GC. Demoralization, anhedonia and grief in patients with severe physical illness. World Psychiatry. 2005;4(2):96-105.

24. Huang J, Fan J, He W, et al. Could intensity ratings of Matsumoto and Ekman's JACFEE pictures delineate basic emotions? A principal component analysis in Chinese university students. Pers Individ Dif. 2009;46(3):331-335.

25. Xu Z, Zhu R, Shen C, et al. Selecting pure-emotion materials from the International Affective Picture System (IAPS) by Chinese university students: a study based on intensity-ratings only. Heliyon. 2017;3(8):e00389.

26. Juslin PN. What does music express? Basic emotions and beyond. Front Psychol. 2013;4(2):596.
27. Biele C, Grabowska A. Sex differences in perception of emotion intensity in dynamic and static facial expressions. Exp Brain Res. 2006;171(1):1-6.

28. O'Connor BP. SPSS and SAS programs for determining the number of components using parallel analysis and Velicer's MAP test. Behav Res Methods Instrum Comput. 2000;32(3):396-402.

29. Arbuckle JA. AMOS User's Guide (V. 3.6). Chicago, IL: Small Waters Corp.; 1997.

30. Lai JS, Nowinski C, Victorson D, et al. Quality-of-life measures in children with neurological conditions: pediatric Neuro-QOL. Neurorehabil Neural Repair. 2012;26(1):36-47.

31. Toyama M, Yamada Y. The relationships among tourist novelty, familiarity, satisfaction, and destination loyalty: beyond the novelty-familiarity continuum. Int J Mark Stud. 2012;4(6):10-18.

32. Barry TJ, Hermans D, Lenaert B, Debeer E, Griffith JW. The eacs: attentional control in the presence of emotion. Pers Individ Dif. 2013;55(7):777-782.

33. Plutchik R. The Psychology and Biology of Emotions. Vol. 2. NewYork, NY: Harper-Collins; 1994.

34. Lindström E, Juslin PN, Bresin R, Williamon A. "Expressivity comes from within your soul": a questionnaire study of music students' perspectives on expressivity. Res Stud Music Educ. 2003;20(1):23-47.

35. Mohn C, Argstatter H, Wilker F-W. Perception of six basic emotions in music. Psychol Music. 2011;39(4):503-517.

36. Hunter PG, Schellenberg EG. Music and emotion. In: Jones MR, Fay RR, Popper AN, editors. Music Perception. New York: Springer; 2010:129-164.

37. Kalawski JP. Is tenderness a basic emotion? Motiv Emot. 2010;34(2): 158-167.

38. Sharman L, Dingle GA. Extreme metal music and anger processing. Front Hum Neurosci. 2015;9:272.

39. Schwartz GE, Weinberger DA, Singer JA. Cardiovascular differentiation of happiness, sadness, anger, and fear following imagery and exercise. Psychosom Med. 1981;43(4):343-364.

40. Hevner K. The affective value of pitch and tempo in music. Am J Psychol. 1937;49(4):621-630.

41. Hu X, Downie JS. Exploring mood metadata: relationships with genre, artist and usage metadata. Paper presented at: International Symposium/ Conference on Music Information Retrieval; September 23-27; 2007; Vienna, Austria.

42. Skowronek J, McKinney MF, de Par SV. A demonstrator for automatic music mood estimation. Paper presented at: International Symposium/ Conference on Music Information Retrieval; September 23-27; 2007; Vienna, Austria.

43. Hu X, Downie JS, Laurier C, Bay M, Ehmann AF. The 2007 MIREX audio mood classification task: lessons learned. Paper presented at: International Symposium/Conference on Music Information Retrieval; September 14-18; 2008; Philadelphia, PA, USA.

44. Kawakami A, Furukawa K, Katahira K, Okanoya K. Sad music induces pleasant emotion. Front Psychol. 2013;4:311.

45. Brown R. Music and language. In: Taylor R, editor. Documentary Report of the Ann Arbor Symposium: Applications of Psychology to the Teaching and Learning of Music. Reston, VA: Music Educators National Conference; 1981:233-265.

46. Kawakami A, Furukawa K, Okanoya K. Music evokes vicarious emotions in listeners. Front Psychol. 2014;5:431.

47. Durà-Vilà G, Littlewood R, Leavey G. Depression and the medicalization of sadness: conceptualization and recommended help-seeking. Int J Soc Psychiatry. 2013;59(2):165-175.

48. Eerola T, Peltola HR. Memorable experiences with sad music-reasons, reactions and mechanisms of three types of experiences. PLoS One. 2016;11(6): e0157444

49. Taruffi L, Koelsch S. The paradox of music-evoked sadness: an online survey. PLoS One. 2014;9(10):e110490. 
50. Peltola H-R, Eerola T. Fifty shades of blue: classification of musicevoked sadness. Music Sci. 2016;20(1):84-102.

51. Juslin PN, Sloboda JA, Deutsch D, Deutsch D. Music and emotion. In: Deutsch D, Deutsch D, editors. The Psychology of Music. San Diego, CA, USA: Elsevier Academic Press; 2013:583-645.

52. Kallinen K. Emotional ratings of music excerpts in the western art music repertoire and their self-organization in the Kohonen neural network. Psychol Music. 2005;33(4):373-393.
53. Juslin PN. Can results from studies of perceived expression in musical performances be generalized across response formats? Psychomusicology. 1997;16(1-2):77-101.

54. Robazza C, Macaluso C, D’Urso V. Emotional reactions to music by gender, age, and expertise. Percept Mot Skills. 1994;79(2):939-944.

55. Lim N. Cultural differences in emotion: differences in emotional arousal level between the East and the West. Integr Med Res. 2016;5(2):105-109.

\section{Publish your work in this journal}

Psychology Research and Behavior Management is an international, peerreviewed, open access journal focusing on the science of psychology and its application in behavior management to develop improved outcomes in the clinical, educational, sports and business arenas. Specific topics covered in the journal include: Neuroscience, memory and decision making; Behavior
Submit your manuscript here: https://www.dovepress.com/psychology-research-and-behavior-management-journal 